Bull. Korean Math. Soc. 52 (2015), No. 2, pp. 557-570

http://dx.doi.org/10.4134/BKMS.2015.52.2.557

\title{
ON ACTION OF LAU ALGEBRAS ON VON NEUMANN ALGEBRAS
}

\author{
Mohammad RAmezanpour
}

\begin{abstract}
Let $\mathbb{G}$ be a von Neumann algebraic locally compact quantum group, in the sense of Kustermans and Vaes. In this paper, as a consequence of a notion of amenability for actions of Lau algebras, we show that $\widehat{\mathbb{G}}$, the dual of $\mathbb{G}$, is co-amenable if and only if there is a state $m \in L^{\infty}(\widehat{\mathbb{G}})^{*}$ which is invariant under a left module action of $L^{1}(\mathbb{G})$ on $L^{\infty}(\widehat{\mathbb{G}})^{*}$. This is the quantum group version of a result by Stokke [17]. We also characterize amenable action of Lau algebras by several properties such as fixed point property. This yields in particular, a fixed point characterization of amenable groups and $H$-amenable representation of groups.
\end{abstract}

\section{Introduction}

A Banach algebra $A$ is called a Lau algebra if the dual space $A^{*}$ of $A$ is a $\mathrm{W}^{*}$ algebra and the identity element of $A^{*}$ is a multiplicative linear functional on $A$. The subject of this large class of Banach algebras originated by Lau in [7]. The class of Lau algebras includes the predual of a Hopf von Neumann algebra, the group algebra $L^{1}(G)$, the Measure algebra $M(G)$, the Fourier algebra $A(G)$ and the Fourier-stieltjes algebra $B(G)$ of a locally compact topological group $G$ as defined in [3]. A Lau algebra $A$ is called left amenable if there exists a state $m \in A^{* *}$ which is invariant under canonical left module action of $A$ on $A^{* *}$; that is, $a \cdot m=\varepsilon(a) m$ for all $a \in A$, where $\varepsilon$ denotes the identity of $A^{*}$ [7]. In the same paper, several properties characterizing left amenable Lau algebras are presented. A fixed point characterization can be found in [12].

Let $\mathbb{G}$ be a von Neumann algebraic locally compact quantum group, in the sense of Kustermans and Vaes [6], and let $L^{1}(\mathbb{G})$ be the convolution quantum group algebra of $\mathbb{G}$. Then $\mathbb{G}$ is called amenable if the Lau algebra $L^{1}(\mathbb{G})$ is left amenable; that is there is a state $m \in L^{\infty}(\mathbb{G})^{*}$ which is invariant under canonical left module action of $L^{1}(\mathbb{G})$ on $L^{\infty}(\mathbb{G})^{*}[15]$. If the Banach algebra $L^{1}(\mathbb{G})$ has a bounded approximate identity, then $\mathbb{G}$ is called co-amenable [1].

Received March 11, 2014; Revised July 10, 2014.

2010 Mathematics Subject Classification. 46L65, 46H25, 22D10, 43A07, 22D15.

Key words and phrases. Hopf von Neumann algebra, locally compact quantum group, Lau algebra, unitary representation, amenability. 
Leptin's theorem asserts that a locally compact qroup $G$ is amenable if and only if the Fourier algebra $A(G)$ has a bounded (by one) approximate identity $[9]$. In the language of locally compact quantum groups, it states that $\mathbb{G}$ is amenable if and only if $\widehat{\mathbb{G}}$, the dual of $\mathbb{G}$, is co-amenable. It is known that, if $\widehat{\mathbb{G}}$ is co-amenable, then $\mathbb{G}$ is amenable $[1$, Theorem 3.2]. The converse implication is known to hold in the discrete case [19]. It is an open problem whether this is true for general locally compact quantum groups.

Let $A$ be a Lau algebra. In this paper we introduce and study a notion of amenability for an action of $A$ on a von Neumann algebra. As we shall see, this concept unifies and generalizes the notions of amenability which have been studied in $[2,7,8,13]$ and [16]. We will show that many results concerning left amenability of Lau algebras have similar analogues for amenability of an action of $A$ on a von Neumann algebra. As a consequence, we show that $\widehat{\mathbb{G}}$ is co-amenable if and only if there is a state $m \in L^{\infty}(\widehat{\mathbb{G}})^{*}$ which is invariant under a left module action of $L^{1}(\mathbb{G})$ on $L^{\infty}(\widehat{\mathbb{G}})^{*}$. This generalizes a result of [17] by Stokke on the Fourier algebra to all locally compact quantum groups. Finally, we give a fixed point property characterizing amenability of an action of $A$ on a von Neumann algebra. In particular, we generalize results of [12], and we also give a fixed point characterization of amenable groups and $H$-amenable representation of groups.

\section{Amenability of actions of Lau algebras}

Throughout this paper, we assume that $A$ is a Lau algebra. In this section we introduce a notion of amenability for an action of $A$ on a von Neumann algebra. And then we discuss some of its equivalent formulations. First, we recall some terminology.

By a left Banach $A$-module, we mean a Banach space $X$ equipped with a bounded (by one) bilinear map from $A \times X$ into $X$, denoted by $(a, x) \mapsto a \cdot x$ such that $a \cdot(b \cdot x)=(a b) \cdot x$ for each $a, b \in A$ and $x \in X$. A right Banach $A$-module is defined similarly. The dual spaces $X^{*}$ of a left Banach $A$-module $X$ becomes a dual right Banach $A$-module with the following module actions

$$
(f \cdot a)(x)=f(a \cdot x) \quad\left(f \in X^{*}, x \in X, a \in A\right) .
$$

For a von Neumann algebra $N$, let $P_{1}\left(N^{*}\right)$ be the set of all states of $N$. We put $P_{1}\left(N_{*}\right)=P_{1}\left(N^{*}\right) \cap N_{*}$. If $\varepsilon_{N}$ denotes the identity element of $N$, then $[10$, Corollary 3.3.4], implies that

$$
P_{1}\left(N_{*}\right)=\left\{\omega \in N_{*} ;\|\omega\|=\varepsilon_{N}(\omega)=1\right\} .
$$

We commence this section with the main definition of the paper.

Definition. Let $N$ be a von Neumann algebra. We say that $A$ acts on $N$ if $N$ is a dual right Banach $A$-module. An element $n \in P_{1}\left(N^{*}\right)$ is called a right invariant $A$-mean on $N$ if

$$
n(y \cdot a)=n(y) \quad\left(y \in N, a \in P_{1}(A)\right) .
$$


The action of $A$ on $N$ is called amenable, if there is a right invariant $A$-mean on $N$.

The following examples show that the above definition unifies and generalizes the notions of amenable and $H$-amenable unitary representation of groups, amenable action of groups on von Neumann algebras and amenable action of Hopf-von Neumann algebras.

Example 2.1. (1) Let $A$ acts on $A^{*}$ with the natural module action. Then, this action is amenable if and only if $A$ is left amenable [7].

(2) Let $\left(\pi, H_{\pi}\right)$ be a continuous unitary representation of a locally compact group $G$, and let $B\left(H_{\pi}\right)$ denote the set of all bounded linear operators on $H_{\pi}$. According to [2], $\pi$ is amenable if there exists a state $m$ on $B\left(H_{\pi}\right)$ such that

$$
m\left(\pi(s) T \pi\left(s^{-1}\right)\right)=m(T)
$$

for all $s \in G$ and $T \in B\left(H_{\pi}\right)$. Also, $\pi$ is called $H$-amenable [17], if there exists a net of unit vectors $\left\{\xi_{\alpha}\right\}$ in $H_{\pi}$ such that

$$
\left\|\pi(s) \xi_{\alpha}-\xi_{\alpha}\right\| \rightarrow 0
$$

uniformly on compact subsets of $G$. If, the Lau algebra $L^{1}(G)$ acts on $B\left(H_{\pi}\right)$, as follows

$$
T \cdot f=\int_{G} f(s) \pi\left(s^{-1}\right) T \pi(s) d s \quad\left(T \in B\left(H_{\pi}\right), f \in L^{1}(G)\right),
$$

then, this action is amenable if and only if $\pi$ is amenable [2, Theorem 3.5]. Moreover, the action of $L^{1}(G)$ on $B\left(H_{\pi}\right)$ which is given by

$$
T \cdot f=T \pi(f) \quad\left(T \in B\left(H_{\pi}\right), f \in L^{1}(G)\right),
$$

is amenable if and only if $\pi$ is $H$-amenable [17, Proposition 3.1].

(3) Let $G$ be a locally compact group and let $N$ be a von Neumann algebra. Suppose that $s \rightarrow \beta_{s}$ is a homomorphism of $G$ into $\operatorname{Aut}(N)$ such that for each $y \in N$, the map $s \rightarrow \beta_{s}(y)$ is $\mathrm{w}^{*}$-continuous, then $N$ is called $G$-amenable [8], if there exists a state $m$ on $N$ such that $\left(\beta_{s}\right)^{*}(m)=m$ for all $s \in G$. Now, define

$$
y \cdot f=\int_{G} f(s) \beta_{s^{-1}}(y) d s \quad\left(y \in N, f \in L^{1}(G)\right) .
$$

Then, this action of $L^{1}(G)$ on $N$ is amenable if and only if $N$ is $G$ amenable [8, Proposition 3.2]; see also [16].

(4) A pair $(\mathfrak{M}, \Gamma)$ is a Hopf-von Neumann algebra if $\mathfrak{M}$ is a von Neumann algebra and $\Gamma: \mathfrak{M} \rightarrow \mathfrak{M} \bar{\otimes} \mathfrak{M}$ is a normal, unital *-homomorphism satisfying $(\iota \otimes \Gamma) \Gamma=(\Gamma \otimes \iota) \Gamma$, where $\bar{\otimes}$ denotes the tensor product of von Neumann algebras. We can define a product on $\mathfrak{M}_{*}$, turning it into a Lau algebra, by

$$
\left(\omega_{1} * \omega_{2}\right)(x)=\left(\omega_{1} \otimes \omega_{2}\right) \Gamma(x),
$$


for all $\omega_{1}, \omega_{2} \in \mathfrak{M}_{*}$ and $x \in \mathfrak{M}$. A left action of $(\mathfrak{M}, \Gamma)$ on a von Neumann algebra $N$ is a normal, injective $*$-homomorphism $\alpha: N \rightarrow$ $\mathfrak{M} \bar{\otimes} N$ such that $(\iota \otimes \alpha) \alpha=(\Gamma \otimes \iota) \alpha$. According to [13], $(N, \alpha)$ is left amenable if there exists a state $m$ on $N$ such that

$$
m((\omega \otimes \iota)(\alpha(y)))=\omega(1) N(y) \quad\left(\omega \in \mathfrak{M}_{*}, y \in N\right) .
$$

The action given by $y \cdot \omega=(\omega \otimes \iota) \alpha(y)$ for all $y \in N$ and $\omega \in \mathfrak{M}_{*}$, defines a dual right $\mathfrak{M}_{*}$-module structure on $N$. Now this action is amenable if and only if $(N, \alpha)$ is left amenable.

As first result, we present the following observation which is often useful. Before, we note that, if $A$ acts on a von Neumann algebra $N$, then $N_{*}$ is a left Banach $A$-module. Moreover, $P_{1}(A) \cdot P_{1}\left(N_{*}\right) \subseteq P_{1}\left(N_{*}\right)$ if and only if $\varepsilon_{N} \cdot a=\varepsilon_{N}$ for each $a \in P_{1}(A)$. Indeed, if $P_{1}(A) \cdot P_{1}\left(N_{*}\right) \subseteq P_{1}\left(N_{*}\right)$ and $\omega \in P_{1}\left(N_{*}\right)$, then $\left(\varepsilon_{N} \cdot a\right)(\omega)=\varepsilon_{N}(a \cdot \omega)=1=\varepsilon_{N}(\omega)$. So, $\varepsilon_{N} \cdot a=\varepsilon_{N}$. Conversely, if $\varepsilon_{N} \cdot a=\varepsilon_{N}$, $a \in P_{1}(A)$ and $\omega \in P_{1}\left(N_{*}\right)$, then $(a \cdot \omega)\left(\varepsilon_{N}\right)=\omega\left(\varepsilon_{N} \cdot a\right)=\omega\left(\varepsilon_{N}\right)=1$. So $P_{1}(A) \cdot P_{1}\left(N_{*}\right) \subseteq P_{1}\left(N_{*}\right)$.

Proposition 2.2. Let $A$ act on a von Neumann algebra $N$ and $\varepsilon_{N} \cdot a=\varepsilon_{N}$ for each $a \in P_{1}(A)$. Then the action of $A$ on $N$ is amenable if and only if there is a non-zero $n \in N^{*}$ such that $a \cdot n=n$ for each $a \in P_{1}(A)$.

Proof. Suppose that $n$ is a non-zero element of $N^{*}$ such that $a \cdot n=n$ for each $a \in P_{1}(A)$. By [10, Theorem 3.3.2], we have $a \cdot n^{*}=n^{*}$ for all $a \in P_{1}(A)$. So we may assume that $n$ is self adjoint. By Jordan decomposition theorem, [10, Theorem 3.3.10], there exists positive linear functionals $n^{+}$and $n^{-}$on $N$ such that $n=n^{+}-n^{-}$and $\|n\|=\left\|n^{+}\right\|+\left\|n^{-}\right\|$. If $a \in P_{1}(A)$, then $n=a \cdot n=a \cdot n^{+}-a \cdot n^{-}$. But $a \cdot n^{+}$and $a \cdot n^{-}$are positive and

$$
\begin{aligned}
\left\|a \cdot n^{+}\right\|+\left\|a \cdot n^{-}\right\| & =\left(a \cdot n^{+}\right)\left(\varepsilon_{N}\right)+\left(a \cdot n^{-}\right)\left(\varepsilon_{N}\right)=n^{+}\left(\varepsilon_{N} \cdot a\right)+n^{-}\left(\varepsilon_{N} \cdot a\right) \\
& =n^{+}\left(\varepsilon_{N}\right)+n^{-}\left(\varepsilon_{N}\right)=\left\|n^{+}\right\|+\left\|n^{-}\right\|=\|n\| .
\end{aligned}
$$

By the uniqueness of decomposition it follows that $a \cdot n^{+}=n^{+}$and $a \cdot n^{-}=n^{-}$. Consequently, if $n^{+} \neq 0$ (say) and $m=n^{+} / n^{+}\left(\varepsilon_{N}\right)$, then $m \in P_{1}\left(N^{*}\right)$ and $a \cdot m=m$ for each $a \in P_{1}(A)$. Therefore, the action of $A$ on $N$ is amenable.

In the next result, left amenable Lau algebras are characterized by the amenability of all their actions. This result covers [2, Theorem 2.2], and [13, Proposition 2.5] for the special cases presented in Example 2.1. First, we recall that a Lau algebra $A$ is left amenable if there is an element $m \in P_{1}\left(A^{* *}\right)$ such that $a \cdot m=m$ for each $a \in P_{1}(A)$. Examples of left amenable Lau algebras include all commutative Lau algebra, $L^{1}(G)$ and $M(G)$ when $G$ is amenable group; see [7] for details.

Proposition 2.3. Let $A$ be a Lau algebra then, $A$ is left amenable if and only if any action of $A$ on a von Neumann algebra which $\varepsilon_{N} \cdot a=\varepsilon_{N}$ for each $a \in P_{1}(A)$, is amenable. 
Proof. Let $m \in P_{1}\left(A^{* *}\right)$ be such that $a \cdot m=m$ for each $a \in P_{1}(A)$. Let $A$ act on a von Neumann algebra $N$ and $\varepsilon_{N} \cdot a=\varepsilon_{N}$ for each $a \in P_{1}(A)$. Fix $\omega \in P_{1}\left(N_{*}\right)$ and define $n: N \rightarrow \mathbb{C}$ by $n(y)=m(\omega \star y)$ where $\omega \star y \in A^{*}$ is defined by $(\omega \star y)(a)=y(a \cdot \omega)$. Then $n \in P_{1}\left(N^{*}\right)$ and

$$
n(y \cdot a)=m(\omega \star(y \cdot a)))=m((\omega \star y) \cdot a)=m(\omega \star y)=n(y)
$$

for all $a \in P_{1}(A)$ and $y \in N$. So the action of $A$ on $N$ is amenable. The converse is clear.

Apply Proposition 2.3 for the actions presented in Example 2.1(2) and (3) we have the next result; compare [2, Theorem 2.2]. First, we recall that a locally compact group $G$ is called amenable if there is a functional $m$ on the dual $L^{\infty}(G)$ of $L^{1}(G)$ such that $m\left(L_{s} f\right)=m(f)$ for all $f \in L^{\infty}(G)$ and $s \in G$, where $L_{s} f(t)=f(s t)$ for all $t \in G$. Examples of amenable groups include all commutative groups and all compact groups; refer to [14] for details.

Corollary 2.4. For a locally compact group $G$ the following are equivalent:

(i) $G$ is amenable.

(ii) every unitary representation of $G$ is amenable.

(iii) every action $s \rightarrow \beta_{s}$ of $G$ on a von Neumann algebra is $G$-amenable.

Proof. The equivalence of (i) and (ii) follows from Proposition 2.3 and this fact that $G$ is amenable if and only if the left regular representation $\left(\lambda, L^{2}(G)\right)$ is amenable [17, Remark 2.3]. Also the equivalence of (i) and (iii) follows from this fact that $G$ is amenable if and only if $L^{\infty}(G)$ is $G$-amenable with respect to the action $s \rightarrow \lambda(s)$.

Next, we characterize amenable actions of $A$ on a von Neumann algebra by the existence of nets of normal states converging to invariance; compare [7, Theorem 4.6], [2, Theorem 3.6], [17, Proposition 3.1] and [13, Lemma 2.7] for the special cases presented in Example 2.1.

Proposition 2.5. Let $A$ act on a von Neumann algebra $N$, then the following are equivalent:

(i) the action of $A$ on $N$ is amenable.

(ii) there is a net $\left\{\omega_{i}\right\}$ in $P_{1}\left(N_{*}\right)$ such that $a \cdot \omega_{i}-\omega_{i} \stackrel{w}{\longrightarrow} 0$ in $N_{*}$, for all $a \in P_{1}(A)$.

(iii) there is a net $\left\{\omega_{i}\right\}$ in $P_{1}\left(N_{*}\right)$ such that $\lim _{i}\left\|a \cdot \omega_{i}-\omega_{i}\right\|=0$, for all $a \in P_{1}(A)$.

Proof. The result follows by a standard argument similar to the Namioka's idea in [11](see also [19, Lemma 3.5]); however, we give a sketch of the proof.

(i) $\Rightarrow$ (ii): Let $n$ be a right invariant $A$-mean on $N$. Choose a net $\left\{\omega_{i}\right\} \subseteq$ $P_{1}\left(N_{*}\right)$ such that $\omega_{i} \rightarrow n$ in the $\mathrm{w}^{*}$-topology of $N^{*}$. Thus $a \cdot \omega_{i}-\omega_{i} \stackrel{w}{\longrightarrow} 0$ for each $a \in A$.

(ii) $\Rightarrow$ (iii): Let $\mathfrak{F}$ be the set of all finite subsets of $P_{1}(A)$ and $\left\{\omega_{i}\right\}_{i \in I}$ be the net in $P_{1}\left(N_{*}\right)$ such that $a \cdot \omega_{i}-\omega_{i} \stackrel{w}{\longrightarrow} 0$ in $N_{*}$, for all $a \in P_{1}(A)$. For 
$F=\left\{a_{1}, a_{2}, \ldots, a_{k}\right\} \in \mathfrak{F}$ consider the Banach space $B_{F}=\sum_{a \in F} N_{*}$ with $\ell^{1}$-norm. Set

$$
x_{F}(\omega)=\left(a_{1} \cdot \omega-\omega, a_{2} \cdot \omega-\omega, \ldots, a_{k} \cdot \omega-\omega\right) \quad\left(\omega \in N_{*}\right) .
$$

Then $x_{F}\left(\omega_{i}\right) \stackrel{w}{\longrightarrow} 0$ in $B_{F}$. So the norm closure of the convex hull of $\left\{x_{F}\left(\omega_{i}\right)\right.$; $i \in I\}$ contains 0 . Hence for any $n \in \mathbb{N}$ there exists a state $\nu_{(F, n)} \in N_{*}$ such that

$$
\left\|a \cdot \nu_{(F, n)}-\nu_{(F, n)}\right\|<\frac{1}{n}
$$

for any $a \in F$. Then new net $\left\{\nu_{(F, n)}\right\}_{(F, n) \in \mathfrak{F} \times \mathbb{N}}$ is a desired one.

(iii) $\Rightarrow(\mathrm{i})$ : It is evident that any $\mathrm{w}^{*}$-cluster point of the net $\left\{\omega_{i}\right\}$, presented in assumption, is a right invariant $A$-mean on $N$. So the action of $A$ on $N$ is amenable.

Proposition 2.6. Let $A$ act on a von Neumann algebra $N$ such that $\varepsilon_{N} \cdot a=\varepsilon_{N}$ for all $a \in P_{1}(A)$ and let $Y$ be a closed sub-module of $N$ with $\varepsilon_{N} \in Y$. If there is a bounded right $A$-module map $P: N \rightarrow Y$ such that $P\left(\varepsilon_{N}\right)=\varepsilon_{N}$, then the action of $A$ on $N$ is amenable if and only if there is an element $n \in Y^{*}$ such that $n\left(\varepsilon_{N}\right)=\|n\|=1$ and $a \cdot n=n$ for each $a \in P_{1}(A)$.

Proof. Let $n \in Y^{*}$ be such that $n\left(\varepsilon_{N}\right)=\|n\|=1$ and $a \cdot n=n$ for each $a \in P_{1}(A)$. Set $m=P^{*}(n)$. Then $m\left(\varepsilon_{N}\right)=1$, and

$$
m(y \cdot a)=n(P(y \cdot a))=n(P(y) \cdot a)=n(P(y))=m(y)
$$

for all $y \in N$ and $a \in P_{1}(A)$. Applying Proposition 2.2, the action of $A$ on $N$ is amenable. For the converse it is enough to restrict the right invariant $A$-mean to $Y$.

Let $\left\{e_{\alpha}\right\}$ be a bounded approximate identity for $A$ and let $A$ act on a von Neumann algebra $N$ such that $\varepsilon_{N} \cdot a=\varepsilon_{N}$ for all $a \in P_{1}(A)$. Put $[N \cdot A]=$ $\overline{\operatorname{Span}}\{y \cdot a ; y \in N, a \in A\}$. Then $[N \cdot A]$ is a self adjoint and closed sub-module of $N$ and $\varepsilon_{N} \in[N \cdot A]$. Define $P: N \rightarrow[N \cdot A]$ by $P(y)=\lim _{\alpha} y \cdot e_{\alpha}$. Then $P\left(\varepsilon_{N}\right)=\varepsilon_{N}$. Moreover, for all $y \in N$ and $a \in A$ we have

$$
P(y \cdot a)=\lim _{\alpha} y \cdot\left(a e_{\alpha}\right)=\lim _{\alpha} y \cdot\left(e_{\alpha} a\right)=P(y) \cdot a .
$$

Thus the next result, which is a generalization of [2, Theorem 3.5] and [13, Lemma 2.7], follows from Proposition 2.6. This result shows that [13, Lemma 2.7 is also valid when $\mathfrak{M}_{*}$ has a bounded approximate identity.

Corollary 2.7. Let $A$ act on a von Neumann algebra $N$ such that $\varepsilon_{N} \cdot a=\varepsilon_{N}$ for all $a \in P_{1}(A)$. If $A$ has a bounded approximate identity, then the action of $A$ on $N$ is amenable if and only if there is an element $n \in[N \cdot A]^{*}$ such that $n\left(\varepsilon_{N}\right)=\|n\|=1$ and $a \cdot n=n$ for each $a \in P_{1}(A)$. 


\section{Application to quantum groups}

Let us start this section by recalling some notation related to locally compact quantum groups. We present a very quick account of this notion which has been adopted from $[5,6]$ and [20]. A locally compact quantum group is a triplet $\mathbb{G}=\left(L^{\infty}(\mathbb{G}), \Gamma, \varphi\right)$, where $\left(L^{\infty}(\mathbb{G}), \Gamma\right)$ is a Hopf von Neumann algebra, and $\varphi$ is a normal faithful semifinite left Haar Weight on $L^{\infty}(\mathbb{G})[5,6]$. Let $L^{2}(\mathbb{G})$ be the Hilbert space associated with $\varphi$. If we denote the Hilbert space tensor product by $\otimes_{2}$, then there exists a left fundamental unitary operator $W \in B\left(L^{2}(\mathbb{G}) \otimes_{2} L^{2}(\mathbb{G})\right)$ implementing the co-multiplication $\Gamma$ via

$$
\Gamma(x)=W^{*}(1 \otimes x) W \quad\left(x \in L^{\infty}(\mathbb{G})\right) .
$$

Let $L^{1}(\mathbb{G})$ be the predual of $L^{\infty}(\mathbb{G})$. The left regular representation $\lambda$ : $L^{1}(\mathbb{G}) \rightarrow B\left(L^{2}(\mathbb{G})\right)$ is defined by

$$
\lambda(\omega)=(\omega \otimes \iota)(W) \quad\left(\omega \in L^{1}(\mathbb{G})\right) .
$$

It is an injective and contractive homomorphism. Setting $L^{\infty}(\widehat{\mathbb{G}})=\{\lambda(\omega): \omega \in$ $\left.L^{1}(\mathbb{G})\right\}^{\prime \prime}$, and put $\widehat{W}=\sigma W^{*} \sigma$, where $\sigma$ denotes the flip map on $L^{2}(\mathbb{G}) \otimes_{2} L^{2}(\mathbb{G})$. Define

$$
\widehat{\Gamma}(y)=\widehat{W}^{*}(1 \otimes y) \widehat{W},
$$

it can be shown that $\widehat{\Gamma}$ is a co-multiplication. One can also define a left Haar weight $\widehat{\varphi}$ on $L^{\infty}(\widehat{\mathbb{G}})$ that $\widehat{\mathbb{G}}=\left(L^{\infty}(\widehat{\mathbb{G}}), \widehat{\Gamma}, \widehat{\varphi}\right)$, the dual quantum group of $\mathbb{G}$, turn it into a locally compact quantum group. Moreover, a Pontryagin duality theorem holds; that is, $\widehat{\widehat{G}}=\mathbb{G}[6]$.

If $G$ is a locally compact group, then $\mathbb{G}_{a}=\left(L^{\infty}(G), \Gamma_{a}, \varphi_{a}\right)$ is a locally compact quantum group, where the co-multiplication is given by $\Gamma_{a}(f)(s, t)=$ $f(s t)$, and $\varphi_{a}$ is integration with respect to a left Haar measure. The dual quantum group $\widehat{\mathbb{G}}_{a}$ of $\mathbb{G}_{a}$ is $\mathbb{G}_{s}=\left(V N(G), \Gamma_{s}, \varphi_{s}\right)$, where $V N(G)$ is the left group von Neumann algebra with co-multiplication $\Gamma_{s}(\lambda(t))=\lambda(t) \otimes \lambda(t)$, and $\varphi_{s}$ is Plancheral weight [18, Definition VII.3.2].

Using the co-multiplication $\Gamma$ the canonical $L^{1}(\mathbb{G})$-bimodule structure on $L^{\infty}(\mathbb{G})$, can be presented by

$$
\omega \cdot x=(\iota \otimes \omega) \Gamma(x) \quad \text { and } \quad x \cdot \omega=(\omega \otimes \iota) \Gamma(x) \quad\left(x \in L^{\infty}(\mathbb{G}), \omega \in L^{1}(\mathbb{G})\right) .
$$

A locally compact quantum group $\mathbb{G}$ is said to be amenable if there exists a state $m \in L^{\infty}(\mathbb{G})^{*}$ which is left invariant; that is $m(\omega \cdot x)=\omega(1) m(x)$ for all $\omega \in L^{1}(\mathbb{G})$ and $x \in L^{\infty}(\mathbb{G})$. It is known that $\mathbb{G}$ is amenable if and only if there exists a right (equivalently, two-sided) invariant mean [15]. Also $\mathbb{G}$ is said to be co-amenable if $L^{1}(\mathbb{G})$ has a bounded left (equivalently, right or two-sided) approximate identity [1, Theorem 3.1$]$. In the two classical cases $\mathbb{G}_{a}$ and $\mathbb{G}_{s}$; $\mathbb{G}_{a}$ is always co-amenable and it is amenable if and only if $G$ is amenable and $\mathbb{G}_{s}$ is always amenable and it is co-amenable if and only if the group $G$ is amenable. 
Let $\mathbb{G}$ be a locally compact quantum group. Before we give the following characterization of co-amenability of $\widehat{\mathbb{G}}$, we should mention that the Lau algebra $L^{1}(\mathbb{G})$ can acts on $L^{\infty}(\widehat{\mathbb{G}})$ by

$$
\widehat{x} \cdot \omega=\widehat{x}(\omega \otimes \iota)(W)=\widehat{x} \lambda(\omega) \quad\left(\widehat{x} \in L^{\infty}(\widehat{\mathbb{G}}), \omega \in L^{1}(\mathbb{G})\right) .
$$

Theorem 3.1. Let $\mathbb{G}$ be a locally compact quantum group. Then the following are equivalent:

(i) $\widehat{\mathbb{G}}$ is co-amenable.

(ii) There is a net $\left\{\widehat{\omega}_{i}\right\}$ in $P_{1}\left(L^{1}(\widehat{\mathbb{G}})\right)$ such that $\lim _{i}\left\|\omega \cdot \widehat{\omega}_{i}-\widehat{\omega}_{i}\right\|=0$ for all $\omega \in P_{1}\left(L^{1}(\mathbb{G})\right)$.

(iii) There is a state $m \in L^{\infty}(\widehat{\mathbb{G}})^{*}$ such that $m(\widehat{x} \cdot \omega)=\omega(1) m(\widehat{x})$ for each $\omega \in L^{1}(\mathbb{G})$ and $\widehat{x} \in L^{\infty}(\widehat{\mathbb{G}})$.

(iv) There is a state $m \in L^{\infty}(\widehat{\mathbb{G}})^{*}$ such that $m(\widehat{\omega} \cdot \widehat{x})=\widehat{\omega}(\widehat{x})$ for each $\widehat{\omega} \in L^{1}(\widehat{\mathbb{G}})$ and $\widehat{x} \in L^{\infty}(\widehat{\mathbb{G}})$.

Proof. It is easy to see (iv) holds if and only if the Banach algebra $L^{1}(\widehat{\mathbb{G}})$ has a left approximate identity consisting of states. By [4, Theorem 2], we conclude that (i) and (iv) are equivalent. In view of Proposition 2.5, it is enough to show that (i) and (ii) are equivalent.

Suppose (i) holds. By [1, Theorem 3.1], there exists a net $\left\{\xi_{i}\right\}$ of unit vectors in $L^{2}(\mathbb{G})$ such that

$$
\left\|W\left(\eta \otimes \xi_{i}\right)-\eta \otimes \xi_{i}\right\|_{2} \rightarrow 0,
$$

for each $\eta \in L^{2}(\mathbb{G})$. Define $\widehat{\omega}_{i} \in P_{1}\left(L^{1}(\widehat{\mathbb{G}})\right)$ to be the restriction of $\omega_{\xi_{i}}$ to $L^{\infty}(\widehat{\mathbb{G}})$. Then, for all unit vector $\eta \in L^{2}(\mathbb{G})$ and all norm one elements $\widehat{x} \in$ $L^{\infty}(\widehat{\mathbb{G}})$, we have

$$
\begin{aligned}
\left|\left(\omega_{\eta} \cdot \widehat{\omega}_{i}\right)(\widehat{x})-\widehat{\omega}_{i}(\widehat{x})\right| & =\left|\left(\omega_{\eta} \otimes \widehat{\omega}_{i}\right)((1 \otimes \widehat{x}) W)-\left(\omega_{\eta} \otimes \widehat{\omega}_{i}\right)(1 \otimes \widehat{x})\right| \\
& =\left|\left(\omega_{\eta} \otimes \widehat{\omega}_{i}\right)((1 \otimes \widehat{x}) W-(1 \otimes \widehat{x}))\right| \\
& =\left|\left\langle(1 \otimes \widehat{x})\left(W\left(\eta \otimes \xi_{i}\right)-\eta \otimes \xi_{i}\right), \eta \otimes \xi_{i}\right\rangle\right| \\
& \leq\left\|W\left(\eta \otimes \xi_{i}\right)-\eta \otimes \xi_{i}\right\| .
\end{aligned}
$$

Hence

$$
\left\|\omega_{\eta} \cdot \widehat{\omega}_{i}-\widehat{\omega}_{i}\right\| \leq\left\|W\left(\eta \otimes \xi_{i}\right)-\eta \otimes \xi_{i}\right\| \rightarrow 0 .
$$

Since $L^{\infty}(\mathbb{G})$ is in standard form, every element of $P_{1}\left(L^{1}(\mathbb{G})\right)$ is equal to the restriction of $\omega_{\eta}$, for some unit vector $\eta \in L^{2}(\mathbb{G})$. Therefore, $\left\|\omega \cdot \widehat{\omega}_{i}-\widehat{\omega}_{i}\right\| \rightarrow 0$ for each $\omega \in P_{1}\left(L^{1}(\mathbb{G})\right)$.

Now assume (ii) holds and that $\left\{\widehat{\omega}_{i}\right\}$ is a net of normal states on $L^{\infty}(\widehat{\mathbb{G}})$ such that $\left\|\omega \cdot \widehat{\omega}_{i}-\widehat{\omega}_{i}\right\| \rightarrow 0$ for each $\omega \in P_{1}\left(L^{1}(\mathbb{G})\right)$. Since $L^{\infty}(\widehat{\mathbb{G}})$ is standardly represented on $L^{2}(\mathbb{G})$, there exist unit vectors $\left\{\xi_{i}\right\}$ in $L^{2}(\mathbb{G})$ such that $\widehat{\omega}_{i}=\omega_{\xi_{i}}$ for all $i$. Thus for each unit vector $\eta \in L^{2}(\mathbb{G})$ we have,

$$
\begin{aligned}
\left|\left\langle W\left(\eta \otimes \xi_{i}\right)-\eta \otimes \xi_{i}, \eta \otimes \xi_{i}\right\rangle\right| & =\left|\lambda\left(\omega_{\eta}\right)\left(\omega_{\xi_{i}}\right)-\omega_{\xi_{i}}(1)\right| \\
& =\left|\left(\omega_{\eta} \cdot \omega_{\xi_{i}}-\omega_{\xi_{i}}\right)(1)\right|
\end{aligned}
$$




$$
\leq\left\|\omega_{\eta} \cdot \omega_{\xi_{i}}-\omega_{\xi_{i}}\right\| \rightarrow 0 .
$$

Since $W$ is unitary, we also have $\left|\left\langle W\left(\eta \otimes \xi_{i}\right)-\eta \otimes \xi_{i}, W\left(\eta \otimes \xi_{i}\right)\right\rangle\right| \rightarrow 0$. These follow that,

$$
\begin{aligned}
\left\|W\left(\eta \otimes \xi_{i}\right)-\eta \otimes \xi_{i}\right\|^{2} \leq & \left|\left\langle W\left(\eta \otimes \xi_{i}\right)-\eta \otimes \xi_{i}, W\left(\eta \otimes \xi_{i}\right)\right\rangle\right| \\
& +\left|\left\langle W\left(\eta \otimes \xi_{i}\right)-\eta \otimes \xi_{i}, \eta \otimes \xi_{i}\right\rangle\right| \rightarrow 0 .
\end{aligned}
$$

So $\widehat{\mathbb{G}}$ is co-amenable, by $[1$, Theorem 3.1].

Let $\mathbb{G}$ be a locally compact quantum group and $C_{0}(\widehat{\mathbb{G}})$ be the reduced $\mathrm{C}^{*}$ algebra associated with $\widehat{\mathbb{G}}$; i.e., $C_{0}(\widehat{\mathbb{G}})={\overline{\lambda\left(L^{1}(\mathbb{G})\right)}}^{\|\cdot\|} ;[6]$. The next result follows from the equivalence of (i) and (iii) in Theorem 3.1 and this fact that if $A \subseteq B$ are $\mathrm{C}^{*}$-algebras and $\varphi$ is a multiplicative functional on $A$, its extension $\psi$ to $B$ as a state satisfies $\psi(a b)=\varphi(a) \psi(b)$ and $\psi(b a)=\psi(b) \varphi(a)$ for $a \in A$ and $b \in B$; see [1, Theorem 3.1].

Corollary 3.2. Let $\mathbb{G}$ be a locally compact quantum group, then $\widehat{\mathbb{G}}$ is coamenable if and only if $1 \in L^{\infty}(\mathbb{G})$ extend to a multiplicative functional on $C_{0}(\widehat{\mathbb{G}})$.

Let $G$ be a locally compact group. By Leptin's theorem, $\widehat{\mathbb{G}}_{a}$ is co-amenable if and only if $G$ is amenable. Thus as a particular case of Corollary 3.2, we obtain the following well-known characterization of amenable groups in terms of the existence of certain states on $V N(G)$; see [17].

Corollary 3.3. A locally compact group $G$ is amenable if and only if there is a state $m \in V N(G)^{*}$ such that $m(T \lambda(f))=m(T) \int_{G} f(s) d s$ for each $f \in L^{1}(G)$ and $T \in V N(G)$.

For a locally compact group $G$, recall that the Fourier algebra $A(G)$ is spanned by functions with compact support in $P(G)$, where $P(G)$ denotes the set of all continuous positive definite functions on $G$; see [3] for details. Since $\widehat{\mathbb{G}}_{s}$ is always co-amenable, as a particular case of Theorem 3.1 and Corollary 3.2 , we have the next result. In this result $e$ denotes the identity of $G$.

Corollary 3.4. For a locally compact group $G$ the following holds.

(i) There is a state $m \in L^{\infty}(G)^{*}$ such that $m(\phi u)=u(e) m(\phi)$ for each $u \in A(G)$ and $\phi \in L^{\infty}(G)$.

(ii) There is a net $\left\{f_{i}\right\}$ in $P_{1}\left(L^{1}(G)\right)$ such that $\lim _{i}\left\|u f_{i}-f_{i}\right\|=0$, for all $u \in A(G) \cap P(G)$ with $u(e)=1$.

\section{A fixed point characterization}

Let $N$ be a von Neumann algebra, in this section we give a fixed point characterization of amenability of an action of $A$ on $N$ in the case where $N_{*}$ is a Banach algebra and the action of $A$ on $N$ is compatible; that is

$$
a \cdot(\omega v)=(a \cdot \omega) v \quad\left(\omega, v \in N_{*}, a \in A\right) .
$$


We begin this section with the following definition.

Definition. Let $X$ be a Banach space we say that $X$ is a left Banach $A-N_{*}$ module if $X$ is a left Banach $A$-module and a left Banach $N_{*}$-module, satisfying

$$
(a \cdot \omega) \cdot x=a \cdot(\omega \cdot x) \quad\left(a \in A, x \in X, \omega \in N_{*}\right) .
$$

It is obvious that $N_{*}$ is a left Banach $A-N_{*}$-module. Let $N_{*}^{e}$ be the Banach algebra obtained by adjoining an identity $e$ to $N_{*}$ : so $\|a+\beta e\|=\|a\|+|\beta|$, and canonically, $N_{*}^{e}=N_{*} \oplus \mathbb{C} e$. The left $A$-module action on $N_{*}$ extends to a left $A$-module action on $N_{*}^{e}$ by taking $a \cdot e=e$ for all $a \in A$. So $N_{*}^{e}$ is a left Banach $A-N_{*}$-module. Moreover if $X$ is a left Banach $A-N_{*}$-module and $Y$ is a Banach space, then the projective tensor product $Y \hat{\otimes} X$ with the module actions given by

$$
a \cdot(y \otimes x)=y \otimes(a \cdot x), \quad \omega \cdot(y \otimes x)=y \otimes(\omega \cdot x)
$$

is a left Banach $A-N_{*}$-module.

Let $X$ be a left Banach $A$ - $N_{*}$-module. For each $a \in A$ and $\omega \in N_{*}$ there are $T_{\omega}, T_{a} \in B\left(X^{* *}\right)$, such that

$$
T_{\omega}(F)=\omega \cdot F \quad \text { and } \quad T_{a}(F)=a \cdot F \quad\left(F \in X^{* *}\right) .
$$

By weak* operator topology on $B\left(X^{* *}\right)$, we mean the locally convex topology of $B\left(X^{* *}\right)$ determined by the family

$$
\left\{p_{F, f} ; F \in X^{* *}, f \in X^{*}\right\}
$$

of seminorms on $B\left(X^{* *}\right)$, where

$$
p_{F, f}(T)=|T(F)(f)| \quad\left(T \in B\left(X^{* *}\right)\right) .
$$

Recall that the operator algebra $B\left(X^{* *}\right)$ can be identified with the dual space $\left(X^{* *} \hat{\otimes} X^{*}\right)^{*}$ of $X^{* *} \hat{\otimes} X^{*}$, in a natural way. In particular, the weak* operator topology of $B\left(X^{* *}\right)$ coincides with the weak* topology of $\left(X^{* *} \hat{\otimes} X^{*}\right)^{*}$ on bounded subsets of $B\left(X^{* *}\right)$. We denote by $\Omega_{X}$ the weak* operator closure of the set

$$
\left\{T_{\omega} ; \omega \in P_{1}\left(N_{*}\right)\right\}
$$

in $B\left(X^{* *}\right)$. Therefore $\Omega_{X}$ is compact in the weak* operator topology of $B\left(X^{* *}\right)$.

Before we give our fixed point property, we need the following lemma.

Lemma 4.1. Let $N$ be a von Neumann algebra such that $N_{*}$ is a Banach algebra and let $A$ act on $N$ with compatible action. Assume that $X$ is a left Banach $A-N_{*}$-module such that there is a bounded $N_{*}$-module map $\pi: X \rightarrow N_{*}^{e}$ and $u \in X$ with $\pi(u)=e$. If there is an element $T \in \Omega_{X}$ such that $T_{a} T=T$ for all $a \in P_{1}(A)$, then the action of $A$ on $N$ is amenable.

Proof. By assumption, there exists a net $\left\{\omega_{i}\right\}$ in $P_{1}\left(N_{*}\right)$ such that $T_{\omega_{i}} \rightarrow T$ in the weak* operator topology of $B\left(X^{* *}\right)$. We therefore have $\left(a \cdot \omega_{i}-\omega_{i}\right) \cdot u \stackrel{w}{\longrightarrow} 0$ in $X$, for all $a \in P_{1}(A)$. Now for $y \in\left(N_{*}^{e}\right)^{*}$

$$
y\left(a \cdot \omega_{i}-\omega_{i}\right)=y\left(\left(a \cdot \omega_{i}-\omega_{i}\right) \pi(u)\right)=y\left(\pi\left(\left(a \cdot \omega_{i}-\omega_{i}\right) \cdot u\right)\right)
$$




$$
=\pi^{*}(y)\left(\left(a \cdot \omega_{i}-\omega_{i}\right) \cdot u\right) \rightarrow 0 .
$$

Which implies that $a \cdot \omega_{i}-\omega_{i} \stackrel{w}{\longrightarrow} 0$ in $N_{*}^{e}$ for all $a \in P_{1}(A)$. Since $\left\{a \cdot \omega_{i}-\omega_{i}\right\}$ is a net in $N_{*}$ we have $a \cdot \omega_{i}-\omega_{i} \stackrel{w}{\longrightarrow} 0$ in $N_{*}$, for all $a \in P_{1}(A)$. Hence, the action of $A$ on $N$ is amenable, by Proposition 2.5.

We are now in a position to give a fixed point characterization of amenable actions of $A$ on a von Neumann algebra, compare [8, Theorem 4.3].

Theorem 4.2. Let $N$ be a von Neumann algebra such that $N_{*}$ is a Banach algebra and let $A$ act on $N$ with compatible action. Then, the following conditions are equivalent:

(i) the action of $A$ on $N$ is amenable.

(ii) for each left Banach $A-N_{*}$-module $X$, there exists $T \in \Omega_{X}$ such that $T_{a} T=T$ for all $a \in P_{1}(A)$.

(iii) there exists $T \in \Omega_{N_{*}^{e}}$ such that $T_{a} T=T$ for all $a \in P_{1}(A)$.

(iv) for each Banach space $Y$, there exists $T \in \Omega_{Y \hat{\otimes} N_{*}^{e}}$ such that $T_{a} T=T$ for all $a \in P_{1}(A)$.

Proof. (i) $\Rightarrow$ (ii): Let $X$ be a left Banach $A$ - $N_{*}$-module. Since the action of $A$ on $N$ is amenable, appealing to Proposition 2.5 , there exists a net $\left\{\omega_{i}\right\} \in P_{1}\left(N_{*}\right)$ such that for all $a \in P_{1}(A)$,

$$
\lim _{i}\left\|a \cdot \omega_{i}-\omega_{i}\right\|=0 .
$$

Since $\left\{T_{\omega_{i}}\right\}$ is contained in $\Omega_{X}$, we may assume that $T_{\omega_{i}} \rightarrow T$, in the weak ${ }^{*}$ operator topology of $B\left(X^{* *}\right)$, for some $T \in \Omega_{X}$. For each $a \in P_{1}(A)$, we therefore have $T_{a} T_{\omega_{i}} \rightarrow T_{a} T$ in the weak* operator topology of $B\left(X^{* *}\right)$. Also, by (4.1) we have

$$
\lim _{i}\left\|T_{a} T_{\omega_{i}}-T_{\omega_{i}}\right\|=\lim _{i}\left\|T_{a \cdot \omega_{i}}-T_{\omega_{i}}\right\| \leq K \lim _{i}\left\|a \cdot \omega_{i}-\omega_{i}\right\|=0,
$$

where $K$ is a constant satisfying $\|a \cdot \omega\| \leq K\|a\|\|\omega\|$ for all $a \in A, \omega \in N_{*}$. It follows that $T_{a} T=T$, and so (ii) holds.

The implications (ii) $\Rightarrow$ (iii) and (ii) $\Rightarrow$ (iv) are clear, since $N_{*}^{e}$ and $A \hat{\otimes} N_{*}^{e}$ are left Banach $A-N_{*}$-modules.

(iii) $\Rightarrow$ (i): By using Lemma 4.1, it is enough to let $\pi$ be the identity map on $N_{*}^{e}$ and $u=e$.

(iv) $\Rightarrow$ (i): Take $g \in Y^{*}$ with $\|g\| \leq 1$ and define a map $\pi: Y \otimes N_{*}^{e} \rightarrow N_{*}^{e}$ by $\pi(y \otimes x)=g(y) x$. Then $\pi$ is a continuous linear map. Since $\|\pi\| \leq 1$, it extends to a norm decreasing $N_{*}$-module map $\pi: Y \hat{\otimes} N_{*}^{e} \rightarrow N_{*}^{e}$. Take $y_{0} \in Y$ with $g\left(y_{0}\right)=1$ and put $u=y_{0} \otimes e$. Therefore, the action of $A$ on $N$ is amenable, by Lemma 4.1.

Let $\left(\pi, H_{\pi}\right)$ be a unitary representation of a locally compact group $G$. In view Example 2.1(2), $f \cdot(u v)=(f \cdot u) v$, for all $f \in L^{1}(G)$ and $u, v \in T C\left(H_{\pi}\right)$, where $T C\left(H_{\pi}\right)$ is the Banach algebra of all trace class operators on $H_{\pi}$. So, as a 
consequence of Theorem 4.2, we have the next result, which gives a fixed point characterization of $H$-amenable unitary representation of groups; see [17].

Corollary 4.3. Let $\left(\pi, H_{\pi}\right)$ be a unitary representation of a locally compact group $G$. Then the following are equivalent:

(i) $\pi$ is $H$-amenable.

(ii) for each left Banach $L^{1}(G)-T C\left(H_{\pi}\right)$-module $X$, there exists $T \in \Omega_{X}$ such that $T_{a} T=T$ for all $a \in P_{1}\left(L^{1}(G)\right)$.

(iii) there exists $T \in \Omega_{T C\left(H_{\pi}\right)^{e}}$ such that $T_{a} T=T$ for all $a \in P_{1}\left(L^{1}(G)\right)$.

(iv) for each Banach space $Y$, there exists $T \in \Omega_{Y \hat{\otimes} T C\left(H_{\pi}\right)^{e}}$ such that $T_{a} T=$ $T$ for all $a \in P_{1}\left(L^{1}(G)\right)$.

In above corollary, if we consider the left regular representation of $G$ we obtain a fixed point characterization for amenability of $G$.

Lemma 4.4. Let $N$ be a von Neumann algebra such that $N_{*}$ is a Banach algebra and let $A$ act on $N$ with compatible action. Assume that $X$ is a left Banach $A-N_{*}$-module such that there is a bounded $N_{*}$-module map $\pi: X \rightarrow N_{*}$ and $u \in X$ with $P_{1}\left(N_{*}\right) \pi(u) \subseteq P_{1}\left(N_{*}\right)$. If there is an element $T \in \Omega_{X}$ such that $T_{a} T=T$ for all $a \in P_{1}(A)$, then the action of $A$ on $N$ is amenable.

Proof. Let $\left\{\omega_{i}\right\}$ be a net in $P_{1}\left(N_{*}\right)$ such that $T_{\omega_{i}} \rightarrow T$ in the weak* operator topology of $B\left(X^{* *}\right)$. By a similar argument which was used in the proof of Lemma 4.1 we can show that $\left\{\omega_{i} \pi(u)\right\}$ is a net in $P_{1}\left(N_{*}\right)$ such that

$$
a \cdot\left(\omega_{i} \pi(u)\right)-\omega_{i} \pi(u) \stackrel{w}{\longrightarrow} 0
$$

in $N_{*}$, for all $a \in P_{1}(A)$. Now Proposition 2.5, implies that the action of $A$ on $N$ is amenable.

Next, we give another fixed point characterization for amenability of actions of $A$ on a von Neumann algebra $N$, in the case where $N_{*}$ is also a Lau algebra, compare [8, Theorem 4.5]. Before, let $D$ be a Lau algebra such that $A$ act on $D^{*}$ with compatible action. Let $1 \leq p \leq \infty$, put $\|(b, v)\|_{p}^{p}=\|b\|^{p}+\|v\|^{p}$ and $\|(b, v)\|_{\infty}=\max \{\|b\|,\|v\|\}$. Then $\left(A \times D,\|\cdot\|_{p}\right)$ is a Banach space which we denote it by $A \times_{p} D$. For $\omega \in D$ and $a \in A$ define

$$
\omega \cdot(b, v)=\left(\varepsilon_{D}(\omega) b, \omega v\right), \quad a \cdot(b, v)=(a b, a \cdot v) .
$$

With this actions $A \times_{p} D$ becomes a left Banach $A$-D-module.

Theorem 4.5. Let $D$ be a Lau algebras such that $A$ act on $D^{*}$ with compatible action. Then, the following conditions are equivalent:

(i) the action of $A$ on $D^{*}$ is amenable.

(ii) for each left Banach $A$-D-module $X$, there exists $T \in \Omega_{X}$ such that $T_{a} T=T$ for all $a \in P_{1}(A)$.

(iii) there exists $T \in \Omega_{D}$ such that $T_{a} T=T$ for all $a \in P_{1}(A)$.

(iv) for each Banach space $Y$, there exists $T \in \Omega_{Y \hat{\otimes} D}$ such that $T_{a} T=T$ for all $a \in P_{1}(A)$. 
(v) for some $1 \leq p \leq \infty$, there exists $T \in \Omega_{A \times{ }_{p} D}$ such that $T_{a} T=T$ for all $a \in P_{1}(A)$.

Proof. According to Theorem 4.2 and Lemma 4.4,

For (iii) $\Rightarrow$ (i) let $\pi$ be the identity map on $D$ and take $u \in P_{1}(D)$.

For (iv) $\Rightarrow$ (i) take $g \in Y^{*}$ with $\|g\| \leq 1$ and let $\pi: A \hat{\otimes} D \rightarrow D$ be such that $\pi(a \otimes \omega)=g(y) \omega$. Take $y_{0} \in Y$ with $g\left(y_{0}\right)=1$ and $v \in P_{1}(D)$ and put $u=y_{0} \otimes v$.

For (v) $\Rightarrow$ (i) define $\pi: A \times{ }_{p} D \rightarrow D$ by $\pi(a, v)=v$. Take $b \in A, v \in P_{1}(D)$ and put $u=(b, v)$.

As an application of Theorem 4.5, we present the following descriptions of left amenability of Lau algebras. The equivalence of (1)-(3) was established by Nasr-Isfahani [12].

Corollary 4.6. For a Lau algebra $A$ the following conditions are equivalent:

(i) the Lau algebra $A$ is left amenable.

(ii) for each left Banach $A$-module $X$, there exists $T \in \Omega_{X}$ such that $T_{a} T=$ $T$ for all $a \in P_{1}(A)$.

(iii) there exists $T \in \Omega_{A}$ such that $T_{a} T=T$ for all $a \in P_{1}(A)$.

(iv) for each Banach space $Y$, there exists $T \in \Omega_{Y \hat{\otimes} A}$ such that $T_{a} T=T$ for all $a \in P_{1}(A)$.

(v) for some $1 \leq p \leq \infty$, there exists $T \in \Omega_{A \times{ }_{p} A}$ such that $T_{a} T=T$ for all $a \in P_{1}(A)$.

Let $\mathbb{G}$ be a locally compact quantum group. In above corollary, if we consider the Lau algebra $L^{1}(\mathbb{G})$ we obtain the next result which gives a fixed point characterization for amenability of $\mathbb{G}$.

Corollary 4.7. For a locally compact quantum group $\mathbb{G}$, the following conditions are equivalent:

(i) $\mathbb{G}$ is amenable.

(ii) for each left Banach $L^{1}(\mathbb{G})$-module $X$, there exists $T \in \Omega_{X}$ such that $T_{a} T=T$ for all $a \in P_{1}\left(L^{1}(\mathbb{G})\right)$.

(iii) there exists $T \in \Omega_{L^{1}(\mathbb{G})}$ such that $T_{a} T=T$ for all $a \in P_{1}\left(L^{1}(\mathbb{G})\right)$.

(iv) for each Banach space $Y$, there exists $T \in \Omega_{Y \hat{\otimes} L^{1}(\mathbb{G})}$ such that $T_{a} T=T$ for all $a \in P_{1}\left(L^{1}(\mathbb{G})\right)$.

(v) for some $1 \leq p \leq \infty$, there exists $T \in \Omega_{L^{1}(\mathbb{G}) \times_{p} L^{1}(\mathbb{G})}$ such that $T_{a} T=T$ for all $a \in P_{1}\left(L^{1}(\mathbb{G})\right.$.

Acknowledgments. The author would like to thanks the referee for his/her useful comments and suggestions.

\section{References}

[1] E. Bédos and L. Tuset, Amenability and co-amenability for locally compact quantum groups, Internat. J. Math. 14 (2003), no. 8, 865-884. 
[2] M. E. B. Bekka, Amenable unitary representations of locally compact groups, Invent. Math. 100 (2003), no. 2, 383-401.

[3] P. Eymard, Làlgèbra de Fourier dùn groupe localement compact, Bull. Soc. Math. France 92 (1964), 181-236.

[4] Z. Hu, M. Neufang, and Z.-J. Ruan, Multipliers on a new class of Banach algebras, locally compact quantum groups, and topological centres, Proc. Lond. Math. Soc. (3) 100 (2010), no. 2, 429-458.

[5] J. Kustermans and S. Vaes, Locally compact quantum groups, Ann. Sci. Éc. Norm. Supér (4) 33 (2000), no. 6, 837-934.

[6] Locally compact quantum groups in the von Neumann algebraic setting, Math. Scand. 92 (2003), no. 1, 68-92.

[7] A. T.-M. Lau, Analysis on a class of Banach algebras with applications to harmonic analysis on locally compact groups and semigroups, Fund. Math. 118 (1983), no. 3, $161-175$.

[8] A. T.-M. Lau and A. L. T. Paterson, Group amenability properties for von Neumann algebras, Indiana Univ. Math. J. 55 (2006), no. 4, 1363-1388.

[9] H. Leptin, Sur làlgèbre de Fourier dùn groupe localement compact, C. R. Math. Acad. Sci. Paris 266 (1968), 1180-1182.

[10] G. J. Murphy, $C^{*}$-algebras and Operator Theory, Academic Press, 1990.

[11] I. Namioka, Folner's conditions for amenable semi-groups, Math. Scand. 15 (1964), $18-28$.

[12] R. Nasr-Isfahani, Fixed point characterization of left amenable Lau algebras, Int. J. Math. Math. Sci. 2004 (2004), no. 61-64, 3333-3338.

[13] M. Ramezanpour and H. R. E. Vishki, Reiter's properties for the actions of locally compact quantum groups on von Neumann algebras, Bull. Iranian Math. Soc. 36 (2010), no. $2,1-17,291$.

[14] V. Runde, Lectures on Amenability, Lecture Notes in Mathematics 1774, SpringerVerlag, Berlin, 2002.

[15] _ Characterizations of compact and discrete quantum groups through second duals, J. Operator theory 60 (2008), no. 2, 415-428.

[16] R. Stokke, Quasi-central bounded approximate identities in group algebras of locally compact groups, Illinois J. Math. 48 (2004), no. 1, 151-170.

[17] _ Amenable representations and coefficient subspaces of Fourier-Stieltjes algebras, Math. Scand. 98 (2006), no. 2, 182-200.

[18] M. Takesaki, Theory of Operator Algebras. I, II, Ency. Math. Applic. 124, 125, Springer Verlag, Berlin, 2002.

[19] R. Tomatsu, Amenable discrete quantum groups, J. Math. Soc. Japan 58 (2006), no. 4, 949-964.

[20] A. Van Daele, Locally compact quantum groups. A von Neumann algebra approach, ArXiv:math.OA/0602212.

School of Mathematics and Computer Science

DAMGHAN UNIVERSITY

P. O. Box 36716, Damghan 41167, Iran

E-mail address: ramezanpour@du.ac.ir, md_ramezanpour@yahoo.com 